\title{
Comparação entre a dor provocada pela facoemulsificação com anestesia tópica e a pela infilttração peribulbar sem sedação
}

\author{
Comparison between the pain induced by phacoemulsification with topical anesthesia \\ and by peribulbar anesthesia without sedation
}

\author{
RobertoPintoCoelho ${ }^{1}$ \\ João Weissheimer' ${ }^{2}$ \\ ErasmoRomão ${ }^{3}$ \\ Antonio Augusto Velascoe Cruz ${ }^{4}$
}

\begin{tabular}{|l|}
\hline RESUMO \\
\hline Objetivo: Comparar a sensação de dor produzida pela realização de \\
facoemulsificação com anestesia tópica com a induzida pela infiltração \\
peribulbar de solução anestésica. Métodos: Usando-se uma escala visual \\
análoga de dor de 10 níveis, mediu-se em 20 pacientes, a dor provocada pela \\
realização de facoemulsificação com anestesia tópica (tetracaína 2\%). A \\
mesma escala foi usada para medir em 21 outros pacientes, a dor provocada \\
pela infiltração peribulbar de solução anestésica (lidocaína a $2 \%$ e bupiva- \\
caína 0,5\%). As infiltrações peribulbares e cirurgias foram feitas pelo \\
mesmo cirurgião. As facoemulsificações foram realizadas com acesso \\
"clear cornea" e implante de lente intra-ocular dentro do saco capsular. Não \\
foi administrada qualquer medicação venosa ou via oral. Os valores de dor \\
nos dois grupos estudados foram comparados pelo teste, não paramétrico, \\
de Mann-Whitney U. Resultados: A distribuição dos valores de dor da \\
facectomia comanestesia tópica variou de 0 a 5, com medianaigual a 2. Com \\
a infiltração peribulbar a distribuição obtida foi mais ampla, de 0 a 7, com \\
medianaigual a 3. O teste de Mann-Whitney U, revelou que o "rank" médio \\
do grupo da cirurgia com anestesia tópica (15,78) foi significantemente \\
diferente do obtido com a infiltração peribulbar (25,98) (p=0,0056). \\
Conclusão: Quando não se emprega sedação, a sensação de dor induzida \\
pela realização da cirurgia da facoemulsificação com anestesia tópica é \\
menor do que a causada pela anestesia peribulbar.
\end{tabular}

Descritores: Extração de catarata; Facoemulsificação; Anestesia local; Dor pós-operatória; Medição da dor; Anestésicos locais/Administração \& dosagem; Dor/fisiopatologia; Estudo comparativo
Trabalho realizado no Departamento de Oftalmologia, e Cirurgia de Cabeça e Pescoço da Faculdade de Medicina de Ribeirão Preto - USP.

${ }^{1}$ Médico assistente do setor de catarata do Hospital de Clínicas da Faculdade de Medicina de Ribeirão Preto da Universidade de São Paulo - USP e Doutor em Oftalmologia pela Faculdade de Medicina de Ribeirão Preto da Universidade de São Paulo - USP.

${ }^{2}$ Ex-residente do Hospital de Clínicas da Faculdade de Medicina de Ribeirão Preto da Universidade de São Paulo - USP.

${ }^{3}$ Professor Associado do Departamento de Oftalmologia, Otorrinolaringologia e Cirurgia de Cabeça e Pescoço da Faculdade de Medicina de Ribeirão Preto da Universidade de São Paulo - USP.

${ }^{4}$ Professor Associado do Departamento de Oftalmologia, Otorrinolaringologia e Cirurgia de Cabeça e Pescoço da Faculdade de Medicina de Ribeirão Preto da Universidade de São Paulo - USP.

Endereço para correspondência: Roberto Pinto Coelho. Hospital das Clínicas - Campus. Av. Bandeirantes, 3900 - Ribeirão Preto (SP) CEP 14049-900

E-mail: rpcoelho@keynet.com.br

Recebido para publicação em 19.05.2003

Versão revisada recebida em 18.06.2004

Aprovação em 18.10.2004

\section{INTRODUÇÃO}

Durante muito tempo a anestesia retrobulbar foi a única modalidade anestésica usada em cirurgias oftalmológicas ${ }^{(1)}$. Em 1986, a anestesia peribulbar foi introduzida no arsenal cirúrgico como uma alternativa segura e efetiva às injeções retrobulbares ${ }^{(2)}$. Entretanto, a segurança da anestesia peribulbar começou a ser questionada quando sérias complicações foram relatadas, incluindo perfuração do globo ocular, hemorragia retrobulbar, trauma do nervo óptico, disfunção da musculatura extra-ocular e oclusões venosas retinianas ${ }^{(3)}$.

Vários tipos de anestesia são atualmente empregados nas facectomias. Dependendo da experiência da equipe cirúrgica, das características emocionais do paciente e do tipo de cirurgia a ser empregada, o cirurgião pode 
utilizar diferentes modalidades anestésicas incluindo retrobulbar, peribulbar, subconjuntival, sub-tenoniana e tópica ${ }^{(4-6)}$.

A aplicação de anestesia tópica nas cirurgias oculares não é um procedimento novo. Já em 1884, Knapp relatava o uso de cocaína a 5\% para a extração de catarata ${ }^{(7)}$. A anestesia tópica ganhou um grande impulso na facectomia, com a introdução da facoemulsificação com incisões corneanas (abordagem "clear cornea"), pois nesse tipo de cirurgia, a manipulação conjuntival, episcleral e muscular é mínima, o que diminui em muito a necessidade de acinesia e analgesia bulbar. Na facoemulsificação com anestesia tópica, nenhum anestésico é introduzido na órbita. Dessa maneira, o olho fica normotenso durante toda a cirurgia e a recuperação visual é extremamente rápida ${ }^{(8)}$. Além disso, a anestesia tópica apresenta baixo índice de complicações e elimina o estigma da agulha e toxicidade sistêmica ${ }^{(8)}$.

Uma crítica importante ao uso da anestesia tópica na realização de cirurgias intra-oculares é a sua menor efetividade analgésica em relação à anestesia peri ou retrobulbar o que geraria maior desconforto durante a cirurgia. Na realidade, é difícil comparar-se a dor induzida por diferentes modalidades cirúrgicas, pois a experiência dolorosa é uma sensação subjetiva, comum a todos pacientes e altamente dependente do estado emocional e cultural de cada indivíduo ${ }^{(9)}$.

Este estudo compara a experiência de dor de pacientes submetidos à cirurgia de facoemulsificação com anestesia tópica sem sedação com a de pacientes que foram submetidos à infiltração anestésica peribulbar.

\section{MÉTODOS}

A população de estudo consistiu de 41 pacientes operados por facoemulsificação, dos quais 20 foram sob anestesia tópica (grupo I) e 21 sob anestesia peribulbar (grupo II). Pacientes com catarata total, instabilidade de fixação, fotofobia intensa, dificuldade de manter os olhos abertos durante tonometria e midríase pobre foram excluídos do trabalho. A formação dos grupos foi aleatória, ou seja, não foi aplicado nenhum critério de seleção que os diferenciassem.

A faixa etária, entre 47 e 79 anos, não diferiu entre os grupos e o valores médios de idade não foram significantemente diferentes (teste t para amostras independentes): grupo I 62,95 anos (desvio padrão $=8,18$ ) e grupo II 68,04 anos (desvio padrão= $12,02)(\mathrm{t}=1,58, \mathrm{p}=0,12)$. A relação homem/mulher também não foi significantemente diferente entre os grupos (12 homens nos dois grupos; Qui-quadrado $=0,33, \mathrm{p}=0,85$ ).

Nos dois grupos não foi empregada sedação, e a midríase foi obtida com uso de fenilefrina e tropicamida 3 gotas antes da cirurgia. Somente nos pacientes do grupo II foi utilizado o baroftalmo após a anestesia para se diminuir a pressão intraocular.

Os pacientes foram orientados tanto a respeito do tipo de anestesia que seria empregado durante a cirurgia e como também acerca do questionário sobre a sensação de dor que seria aplicado após o procedimento. A anestesia tópica consistiu de 3 ou 4 gotas de colírio de cloridrato de proximetacaína a $0,5 \%$ (Anestalcon ${ }^{\circledR}$ ) administradas antes da cirurgia com intervalo de 5 minutos entre as gotas. A anestesia peribulbar consistiu na administração de $6 \mathrm{ml}$ de uma mistura de partes iguais de lidocaína $2 \%$ sem adrenalina e bupivacaína $0,5 \%$. A injeção anestésica era feita 15 minutos antes da cirurgia no quadrante ínfero-temporal.

Todos os pacientes foram monitorados com cardioscópio e mantido acesso venoso. As cirurgias foram realizadas pelo mesmo cirurgião de maneira idêntica nos dois grupos. A incisão foi "clear cornea" temporal superior de $2,75 \mathrm{~mm}$ e a paracentese com lâmina de 15 graus. A capsulorexe foi contínua e curvilínea e para a extração da catarata foi utilizado um facoemulsificador marca Alcon, modelo Legacy $20000^{\circledR}$, utilizando-se a técnica "stop-and-chop", seguida da irrigação e aspiração dos restos corticais. Para a introdução de uma lente dobrável, a incisão foi alargada para 3,2 mm dentro do saco capsular. Não foram utilizadas suturas e injeção subconjuntival de antibióticos ou antiinflamatórios no ato cirúrgico. $\mathrm{O}$ tempo cirúrgico não foi quantificado com precisão em todos os pacientes, mas foi estimado em cerca de 20 minutos para a maioria dos pacientes.

Cerca de 15 minutos após as cirurgias, os pacientes do grupo I foram questionados sobre a dor que sentiram durante o procedimento e os do grupo II sobre a dor provocada pela anestesia peribulbar. Em ambos os casos, a sensação de dor foi medida por meio de uma escala análoga visual que possui numeração de 0 - 10 sendo 0 (ausência de dor) e 10 (dor inimaginável $)^{(10)}$. Se o paciente não fosse capaz de ver a escala, o valor da sensação de dor era obtido verbalmente. A comparação da dor entre os dois grupos foi feita por estatística nãoparamétrica através do teste Mann-Whitney U para medianas de amostras independentes.

O estudo foi aprovado previamente pelo comitê de ética medica do Hospital das Clínicas de Ribeirão Preto.

\section{RESULTADOS}

As distribuições dos valores de dor obtidos nos dois grupos são mostradas nas figuras 1 e 2 . O teste de MannWhitney U mostrou que a dor induzida pela anestesia peribulbar ("rank" médio de 25,98) foi significantemente maior do que a provocada pela cirurgia com anestesia tópica ("rank" médio de 15,78$), p=0,0056$.

\section{DISCUSSÃO}

As principais vantagens da cirurgia de catarata sem anestesia retro ou peribulbar são a rápida recuperação da visão e a eliminação das complicações associadas à introdução de uma agulha na órbita (hemorragia retrobulbar, hematoma palpebral, lesão do nervo óptico, perfuração ocular, diplopia e parada respiratória) $)^{(2-3)}$.

A maior desvantagem da facectomia com anestesia tópica 


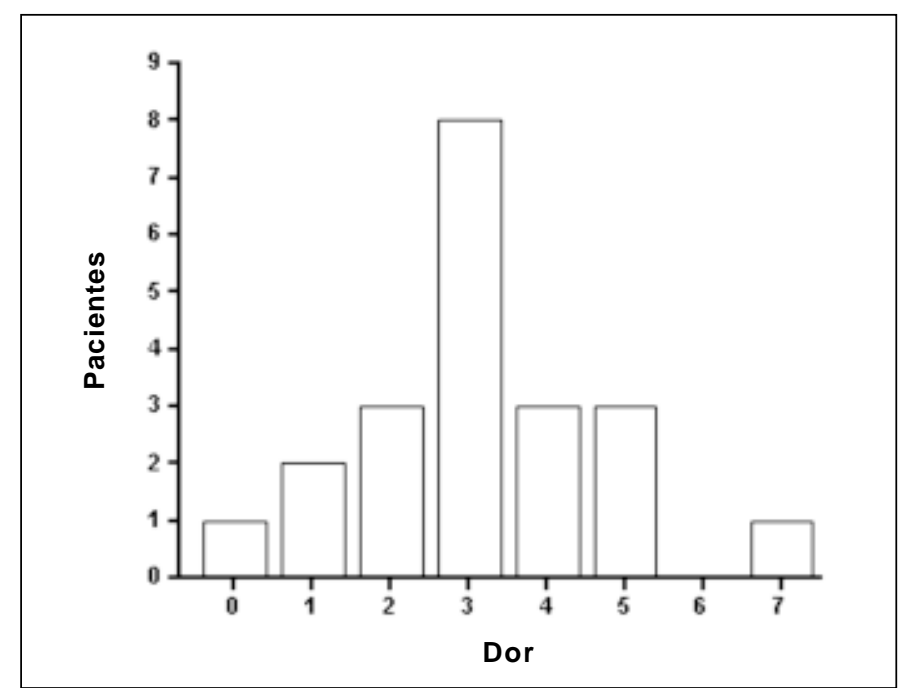

Figura 1 - Distribuição dos valores de dor obtidos com emprego de uma escala análoga de 10 níveis em uma amostra de 21 pacientes após infiltração peribulbar

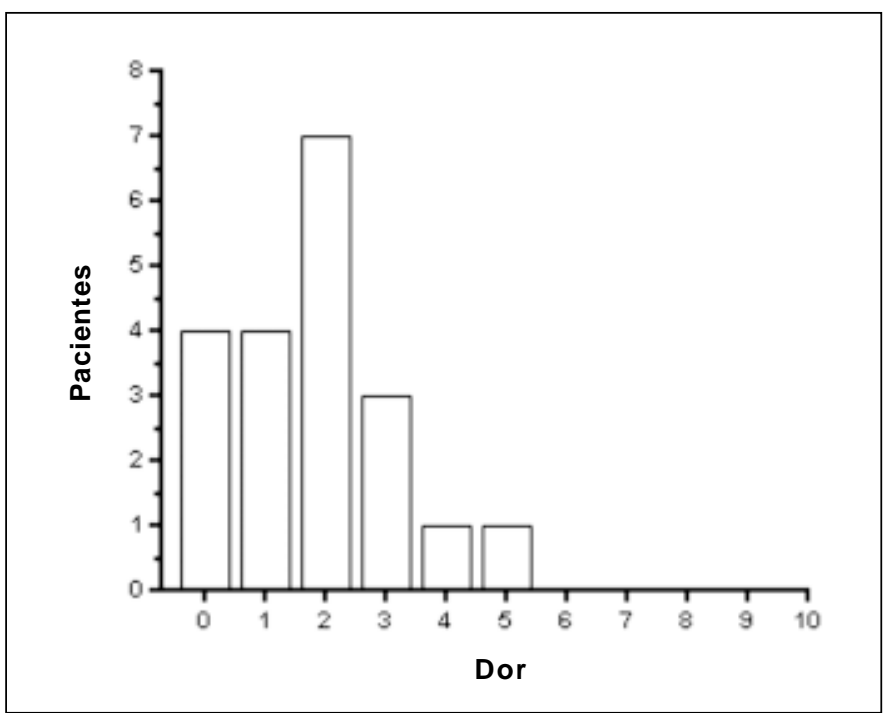

Figura 2 - Distribuição dos valores de dor obtidos com emprego de uma escala análoga de 10 níveis em uma amostra de 20 pacientes após facectomia com anestesia tópica

é a necessidade de maior cooperação do paciente e a mobilidade ocular e palpebral durante a cirurgia. O risco de perda da colaboração pode ser diminuído com uma seleção cuidadosa do paciente. Movimentos oculares inadvertidos e pressão para o fechamento das pálpebras podem ser avaliadas durante o exame de tonometria e eco-biometria ${ }^{(10-11)}$. Comunicação com o paciente é de vital importância para o sucesso com anestesia tópica. Eles devem ser informados que não poderão mover seus olhos durante a cirurgia e que podem sentir alguma sensação durante a cirurgia.

Embora pacientes submetidos à anestesia tópica tolerem bem o procedimento cirúrgico ${ }^{(12-14)}$, a experiência com esta técnica mostra que a luz do microscópio e certos passos cirúrgicos estão associados a desconforto. Esses passos incluem a manipulação da íris, a expansão do globo com a irrigação, e o implante da lente intra-ocular ${ }^{(10-11)}$.

Pacientes ansiosos e que não colaboram não são, obviamente, bons candidatos à cirurgia com anestesia tópica. No presente trabalho, esses pacientes foram excluídos do estudo. Ou seja, não houve nenhum viés no sentido desse tipo de paciente estar em maior número no grupo operado com anestesia peribulbar. Como a formação dos grupos foi aleatória e a distribuição da idade, sexo e nível socioeconômico foi similar, acreditamos que os resultados não tenham sido influenciados por uma distribuição desigual do componente emocional.

A sensação de dor não é simplesmente o resultado da transmissão linear do dano tecidual real ou potencial, mas sim um processo dinâmico que envolve interações contínuas entre sistemas neuronais complexos. Nesse processo, intervêm todo o substrato de experiências cognitivas passadas do sujeito, sua cultura e o seu grau de ansiedade ${ }^{(10)}$. Na origem dessa sensação estão implicados vários fatores, tais como a temperatura do anestésico, a velocidade de injeção, a retirada da agulha, a presença de vasoconstrictor, de conservante e do $\mathrm{pH}$ do anestésico ${ }^{(10)}$.

\section{CONCLUSÃo}

A dor induzida pela facectomia com anestesia tópica é mais difícil de ser caracterizada qualitativamente, mas os resultados do presente estudo mostram que ela é bem menor que a induzida pela anestesia peribulbar. Assim, em pacientes selecionados e cooperativos a facectomia sem sedação e com anestesia tópica é o procedimento que reúne menos riscos para os pacientes com o melhor nível de conforto que a cirurgia com infiltração peribulbar.

\section{ABSTRACT}

Purpose: To compare pain sensation induced by phacoemulsification with topical anesthesia with that by peribulbar anesthesia, without sedation. Methods: Using a 10-level visual pain analogue scale, the pain induced by phacoemulsification with topical anesthesia ( $2 \%$ tetracaine drops) was measured in 20 patients. The same scale was used to measure the pain induced by peribulbar anesthesia (2\% lidocaine and $0.5 \%$ bupivacaine) in 21 patients. The same surgeon performed peribulbar infiltrations and surgeries. The phacoemulsifications were done using clear corneal approach followed by in-the-bag intraocular lens implantation. Pain comparison between the two groups was performed by the non-parametrical U Mann-Whitney test. Results: The distribution of the pain scores of the patients who underwent cataract extraction with topical anesthesia ranged from 0 to 5 (median value $=2$ ). The scores of the patients who underwent peribulbar infiltration ranged from 0 to 7 (median value $=3$ ). The mean rank of the group of patients who had surgery with topical 
anesthesia (15.78) was significantly different from the mean rank of the patients who received peribulbar anesthesia (25.98) $(\mathrm{p}=0.0057)$. Conclusion: Without sedation, the pain induced by phacoemulsification with topical anesthesia was milder than the pain induced by peribulbar infiltration.

Keywords: Cataract extraction; Phacoemulsification; Anesthesia, local; Pain, postoperative; Pain measurement; Anesthetics, local/administration \& dosage; Pain/physiopathology; Comparative study

\section{REFERÊNCIAS}

1. Atkinson WS. Retrobulbar injection of anesthetic within the muscular cone (cone injection). Arch Ophthalmol. 1936;16:494-503.

2. Davis DB II, Mandel MR. Posterior peribulbar anesthesia: an alternative to retrobulbar anesthesia. J Cataract Refract Surg. 1986;12(2):182-4.

3. Morgan CM, Schatz H, Vine AK, Cantrill HL, Davidorf FH, Gitter KA, et al. Ocular complications associated with retrobulbar injections. Ophthalmology. 1998;95(5):660-5.

4. Duguid IG, Claoné CM, Thamby-Rajah Y, Allan BD, Dart JK, Steele AD. Topical anesthesia for phacoemulsification surgery. Eye.1995;9(Pt 4):456-9. Comment in: Eye. 1996;10(Pt 5):650.
5. Gabow HB. Topical anesthesia for cataract surgery. Eur J Implant Refract Surg. 1993;5(1):20-4.

6. Stevens JD. A new local anesthesia for cataract extraction by one quadrant sub-Tenon's infiltration. Br J Ophthalmol. 1992;76(11):670-4. Comment in: Br J Ophthalmol. 1994;78(3):238.

7. Knapp H. On cocaine and its use in ophthalmic and general surgery. Acta Ophthalmol. (old series) 1884;130:402-48.

8. Kershner RM. Topical anesthesia for small incision self-sealing cataract surgery. A prospective evaluation of the first 100 patients. J Cataract Refract Surg. 1993;19(2):290-2. Comment in: J Cataract Refract Surg. 1997;23(10):1435.

9. Revill SI, Robinson JO, Rosen M, Hogg MI. The reliability of a linear analogue scale for evaluating pain. Anaesthesia. 1976;31(9):1191-8.

10. Melzack R. Psychological aspects of pain. Implications for neural blockade. In: Cousins MJ, Bridenbaugh PO. Neural blockade in clinical anesthesia and management of pain. Philadelphia: Lippincott Williams \& Wilkins; 1998. p.781-92.

11. Fraser SG, Siriwadena D, Jamieson H, Girault J, Bryan SJ. Indicators of patient suitability for topical anesthesia. J Cataract Refract Surg. 1997;23(5):781-3.

12. Mathew MR, Webb LA, Hill R. Surgeon experience and patient comfort during clear corneal phacoemulsification under topical local anesthesia. J Cataract Refract Surg. 2002; 28(11):1977-81.

13. Zafirakis P, Voudouri A, Rowe S, Livir-Rallatos G, Livir-Rallatos C, Canakis C, et al. Topical versus sub-Tenon's anesthesia without sedation in cataract surgery. J Cataract Refract Surg. 2001;27(6):873-9.

14. Kallio H, Uusitalo RJ, Maunuksela EL. Topical anesthesia with or without propofofol sedations versus retrobulbar/peribulbar anesthesia for cataract extraction. J Cataract Refract Surg. 2001;27(9):1372-9.

\title{
FÓRUM 2005 DO INSTITUTO PENIDO BURNIER
}

\author{
2 a 4 de Junho de 2005 \\ The Royal Palm Plaza \\ Campinas - SP
}

\section{Tema: Cirurgia da Catarata}

\author{
IN FO RMAÇÕ ES: Tel.: (19) 3232-5866 - Ramal 2147 \\ Fax: (19) 3233-4492 \\ E-mail: penido@ penidoburnier.com.br
}

